\title{
Fenestrated thoracic endovascular aortic repair for zone 2 lesions: Not just basic blocking and tackling
}

\author{
Mario Gaudino, MD, and Leonard N. Girardi, MD
}

From the Department of Cardiothoracic Surgery, Weill Cornell Medical College, New York, NY.

Disclosures: Authors have nothing to disclose with regard to commercial support.

Received for publication Sept 20, 2017; accepted for publication Sept 26, 2017; available ahead of print Oct 26, 2017.

Address for reprints: Mario Gaudino, MD, Department of Cardiothoracic Surgery, Weill Cornell Medical College, 525 E 68th St, New York, NY (E-mail: mfg9004@med.cornell.edu).

J Thorac Cardiovasc Surg 2018;155:494-5

$0022-5223 / \$ 36.00$

Copyright (c) 2017 by The American Association for Thoracic Surgery

https://doi.org/10.1016/j.jtcvs.2017.09.118

In this issue of the Journal, Canaud and colleagues ${ }^{1}$ present $^{-}$ the results of their 3-year experience with using a homemade fenestrated stent graft to repair zone 2 aortic lesions. Canaud and colleagues ${ }^{1}$ modified a commercially available stent graft on the basis of preoperative computed tomographic images to create a fenestration that would allow perfusion to the left subclavian artery (LSA) after deployment. Technical success was achieved in $100 \%$ of the study population. Not surprisingly, $12.5 \%$ of the patients required reintervention for endoleaks within a mean follow-up of 13 months. An additional patient with an endoleak is currently being observed. One patient had a stroke. Pretty good stuff, but is this a procedure ready for more widespread application?

Canaud and colleagues ${ }^{1}$ are to be commended for enrolling a very limited number of patients in an institutional review board-approved protocol. Although one could argue that the results would have been cleaner with the exclusion of those without true zone 2 pathology ( 9 acute, complicated type B dissections), 100\% technical success and no mortality is enviable. There are significant nuances about the planning and execution of this procedure, however, that go beyond basic thoracic endovascular aortic repair blocking and tackling.

Most importantly, Canaud and colleagues ${ }^{1}$ themselves introduced significant modifications to a commercially available device. They withdrew the stent graft from its sheath and utilized cautery to create the fenestration at the predetermined location of the LSA. They then reinforced the fenestration by hand sewing a nitinol wire around the opening, in the hope that this would improve sealing the fenestration to the native aortic wall. The stent graft was then returned to the sheath for deployment and placed into the arch after an undisclosed number of repositionings, rotations, and angiograms to assure proper orientation of the fenestration. A number of patients required placement of a second device into the LSA to avoid a type III endoleak.

As Canaud and colleagues ${ }^{1}$ appropriately highlighted in their discussion, there are some major technical questions that can only be answered with rigorous follow-up of this

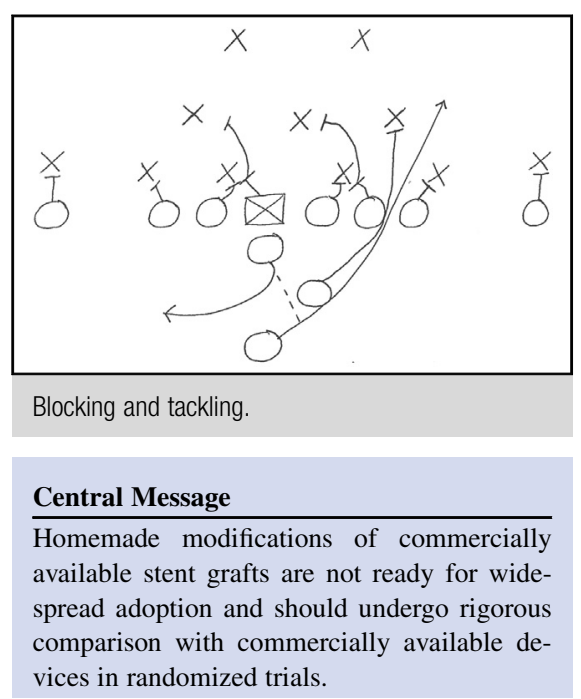

See Article page 488

study cohort. What is the impact of the fenestration on the ring stability of the stent graft? What is the stability of the extra nitinol reinforcement? Will this modification increase the potential for branch stent collapse or breakage that could introduce significant late type III endoleaks? Will fabric durability be affected by the imperfect use of handheld cautery to create the fenestration? What negative events are brought on by the need for additional manipulation of a large device within a diseased aortic arch? Will any of our industry sponsors go along with this (we already know the answer), and what will our colleagues in the legal profession (on both sides of the field) think of this modified, homemade device?

Clearly, LSA coverage during thoracic endovascular aortic repair is less than desirable, and guidelines exist to help us decide when and how we might consider subclavian revascularization during endovascular aortic repair. ${ }^{2} \mathrm{~A}$ carotid-to-subclavian bypass or translocation and "simple" thoracic endovascular aortic repair is not totally endovascular but it does get the job done in what is supposed to be a "high-risk" population (remember the Seahawks throwing the ball on the goal line two Super Bowls ago?). Premanufactured fenestrated stent grafts already exist, with a myriad of great vessel configurations that fit an overwhelming majority of arch anatomy. ${ }^{3}$ This homemade approach is clearly not ready for widespread adoption and should undergo rigorous comparison with these commercially available devices in a randomized trial. 


\section{References}

1. Canaud L, Morishita K, Gandet T, Sfeir J, Bommart S, Alric P, et al. Homemade fenestrated stent-graft for thoracic endovascular aortic repair of zone 2 aortic lesions. J Thorac Cardiovasc Surg. 2018;155:488-93.

2. Matsumura JS, Lee WA, Mitchell RS, Farber MA, Murad MH, Lumsden AB, et al; Society for Vascular Surgery. The Society for Vascular Surgery practice guide- lines: management of the left subclavian artery with thoracic endovascular aortic repair. J Vasc Surg. 2009;50:1155-8.

3. Yokoi Y, Azuma T, Yamazaki K. Advantage of a precurved fenestrated endograft for aortic arch disease: simplified arch aneurysm treatment in Japan 2010 and 2011. J Thorac Cardiovasc Surg. 2013;145(3 Suppl): S103-9. 